\title{
Decreased expression of eukaryotic initiation factor $3 f$ is an adverse prognostic factor for stage I-III gastric cancer
}

\author{
Guanghua Li ${ }^{4 *}$, Na Wang ${ }^{1}$, Chuanjin Sun ${ }^{2}$ and Bo Li ${ }^{3}$
}

\begin{abstract}
Background: It has been demonstrated that elF3f expression is significantly decreased in many human cancers, a fact which plays an important role in human cancer. However, the expression of elF3f in gastric cancer (GC) is not well understood to date. Therefore, the aim of this study is to detect the expression of elF3f in GC.

Methods: The expression of elF3f was examined by immunohistochemistry in tissues with stage I to III GC and adjacent non-cancerous tissues (ANCT) of 195 gastrectomy specimens; clinicopathological results, including survival, were analyzed.

Results: The positive expression rate of elF3f was significantly higher in ANCT tissues than in GC. elF3f levels were correlated with more advanced tumor stages and likelihood of recurrence (all $P<0.05$ ). The Kaplan-Meier survival curves indicated that decreased expression of elF3f could serve as a prognosis marker for poor outcome of GC patients $(P=0.04)$.

Conclusions: elF3f may play an important role in recurrence, thus representing a promising predictive marker for the prognosis of $\mathrm{GC}$.
\end{abstract}

Keywords: Biomarker, elF3f, Gastric cancer, Prognosis

\section{Background}

From a global perspective, gastric cancer (GC) is the fourth most common malignant tumor and the second cause of cancer-related death, accounting for an estimated 989,600 new cases and 738,000 deaths in 2008 [1-3]. In the past several decades, intensive efforts have been made to identify tools to improve prognostication of gastric cancer; miR-21, miRNA-106a, and miR-143, as well as miR-203 are considered to be promising novel biomarkers for GC [4]. However, except for the cancer staging, upon which adjuvant treatment depends, definite predictive factors for the prognosis of GC are relatively scarce [5-8]. Thus, finding new prognostic tools, such as biomarkers, for the accurate diagnosis of GC is of vital importance to improve the therapeutic effect and prolong the survival of patients.

\footnotetext{
* Correspondence: chuanjinsun@126.com

${ }^{4}$ Department of General Surgery, The Second Hospital of Shandong

University, 247th Beiyuan Avenue, Jinan 250200, China

Full list of author information is available at the end of the article
}

The eukaryotic initiation factor $3 f$ (eIF3f) is the p47 subunit of the multi-subunit eIF3 complex and plays an important role in translation initiation [9-11]. Although the role of eIF3f in the eIF3 complex has not been defined [12-14], shut-off experiments in Schizosaccharomyces pombe showed that in a long-term period, eIF3f was essential for viability [15]. The translation system indicated that, in mammalian cells, eIF3f was a negative regulator of translation, which played an important role in human cancer [12,16]. A study by Marchione et al. showed that the $f$ subunit of eIF3 represented a promising candidate molecule to use for bio-therapeutic applications [17]. Previous studies have identified eIF3f as a protein involved in apoptotic signaling as a negative regulator of translation and have also demonstrated that eIF3f expression significantly decreased in many human cancers, and thus played an important role in human cancer $[11,16,18,19]$. Shi et al. reported that eIF3f was downregulated in several human tumors, and that its overexpression inhibited cell proliferation in melanoma 
and pancreatic cancer cells [11]. A recent study by Higareda-Mendoza and Pardo-Galván also showed that eIF3f was essential for A549 cell proliferation and its absence induced the cell to enter apoptosis [20].

Together, these previous findings suggest that eIF3f may be involved in the regulation of cell growth and proliferation and contributes to tumorigenesis; however, its role in GC remains unclear. Thus, in order to shed light into the expression of eIF3f in GC, we correlated eIF3f expression results with the clinicopathologic characteristics and prognosis of GC patients. We conducted experiments to characterize the localization of eIF3f by immunohistochemistry, aiming to find a new prognostic marker for GC and new diagnosis and treatment strategies.

\section{Methods}

\section{Patient samples}

A review of surgical specimens at the The Second Hospital of Shandong University between November 2007 and March 2009 yielded 195 cases of GC patients who had undergone curative surgery. The mean age of the patients was 62 years (range, 31-90 years). The median follow-up period was 38 months (range: 3-54 months).
The TNM stage of tumors was assessed according to the 7th ed. of the TNM Classification of GC [21]. The Her-2/ neu overexpression was detected by postoperative fluorescence in situ hybridization examination. No patient underwent radiotherapy or chemotherapy before operation.

For immunohistochemical staining, the paraffin-embedded tissue sections were obtained from 195 cases of radical resection of GC. The fragments were fixed in neutral formol. All the cases were reviewed by a pathologist to confirm the malignancy. The investigation was approved by the ethics committee of the medical faculty and written informed consents were obtained from all patients.

\section{Immunohistochemistry}

The polyclonal antibody (ab64177) to eIF3f was bought from Abcom (Cambridge, UK). The paraffin-embedded tissue blocks were sectioned in 3 to $4 \mathrm{~mm}$ slices and placed on Anti slides. After de-waxing and hydration, the slides were rinsed in phosphate-buffered saline (PBS) and blocked for $10 \mathrm{~min}$ with $3 \%$ hydrogen peroxide to deprive the endogenous peroxidase activity. After antigen retrieval with the use of a microwave, the specimens

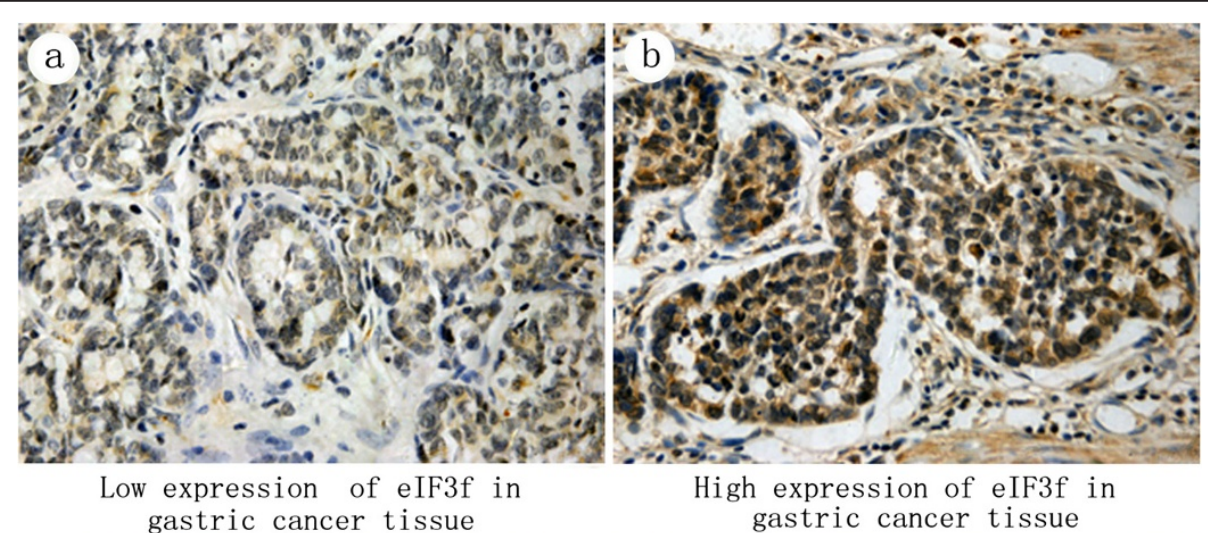

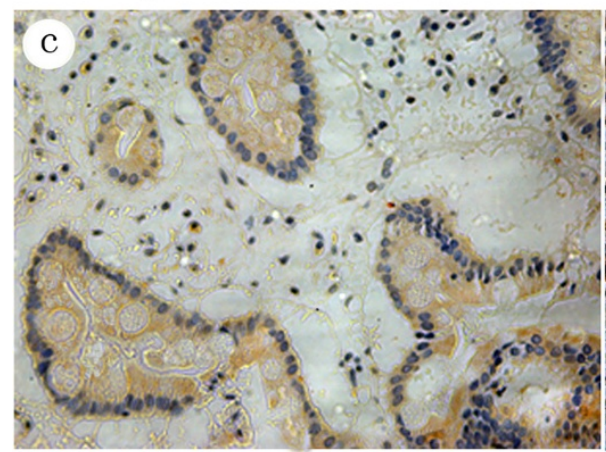

Low expression of eIF3f in adjacent noncancerous tissue

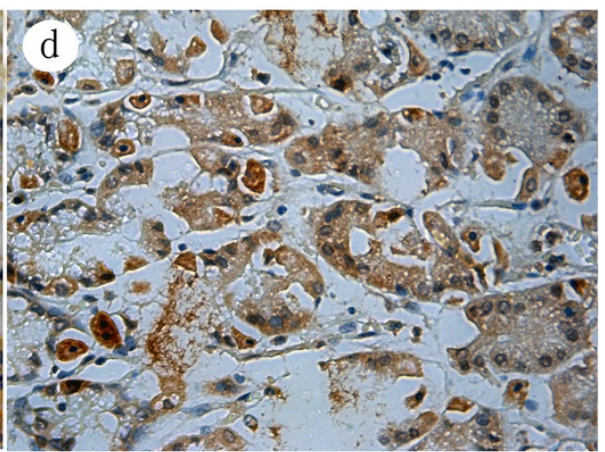

High expression of eIF3f in adjacent noncancerous tissue

Figure 1 Immunohistochemical findings of the degree of expressions of elF3f in gastric cancer (GC) tissue and adjacent non-cancerous tissue (ANCT). elF3f was localized in the cytoplasm at different levels and percentages. (a) and (b) show elF3f staining in GC tissue: low expression (a) and high expression (b) (magnification $\times 400)$. (c) and (d) show elF3f staining in ANCT: low expression (c) and high expression (d) (magnification $\times 400$ ). 
were incubated with the anti eIF3f MAb (diluted 1:100 in $\mathrm{PBS}$ ) at $37^{\circ} \mathrm{C}$ for 1.5 hours. The sections were then washed $3 \times 3 \mathrm{~min}$ in PBS and incubated with 1 and $2 \mathrm{Re}-$ agent of PV9000 Mouse/Rabbit hypersensitivity two-step immunohistochemical Kit (Beijing fir Jinqiao, Beijing, China) for a total of $60 \mathrm{~min}$ at $37^{\circ} \mathrm{C}$ in a humid chamber. The sections were washed $3 \times 3$ min with PBS, followed by the addition of diaminobenzidine as a chromogen for 3 to $5 \mathrm{~min}$, which was strictly controlled under a microscope. Antibodies were optimized using a positive control tissue according to the manufacturer's instructions. In negative controls, the primary antibody was replaced with PBS. The remaining procedures were performed in parallel with other specimens. Each slide was scored in a blinded fashion by two pathologists according to the manufacturer's recommended criteria at $\times 100$ and $\times 200$ magnification. The overall percentage of positive cells on an immunostained section was determined according to the pattern of intracellular localization. The extent and pattern of the eIF3f-specific immunostaining within a tissue section were determined by the percentage of cells with cytoplasm staining. The immunostaining was read in a semiquantitative manner. Three visual fields were examined randomly and the rate of positive cells was divided into less than $5 \%$ (score 0 ), $6 \%$ to $25 \%$ (score 1 ), $26 \%$ to $50 \%$ (score 2), $51 \%$ to $75 \%$ (score 3), and more than $75 \%$ (score 4 ). The staining intensity can be divided into three grades: no staining (score 0 ), slightly yellowish (score 1), brownish yellow (score 2), and dark brown (score 3). The multiplication of the two were graded as follows: 0 (score 0 ), $1+$ (score 1-4), $2+$ (score 5-8), and $3+$ (score 9-12). Intensity scores of 0 or $1+$ were designated as low expression and $2+$ or $3+$ were designated as high expression.

\section{Statistical analysis}

Differences between groups were analyzed using a Student's $t$-test for continuous variables and a $x^{2}$ test or Fisher's exact test for proportions. The overall survival rate was estimated by the Kaplan-Meier method. Univariate analyses (Cox proportional hazard regression models) were also performed to assess the prognostic value of nucleolin expression and other clinicopathological features. We utilized SAS 9.2 software system for statistical analysis.

\section{Results}

elF3f expression and clinicopathological characteristics

From the 195 cases, 142 men and 53 women all accepted radical operation of GC, including 116 primary and 79 recurrent GC patients. Pathologically, all of the 195 cases were adenocarcinoma, which covered 12 well differentiated, 27 moderate differentiated, 156 low differentiated, and 4 signet-ring cell carcinoma. For all specimens, 31 cases were demonstrated as Her-2/neu-positive.

\section{elF3f expression in gastric cancer and ANCT}

Immunohistochemical staining for eIF3f showed a cytoplasm staining pattern in both GC (Figure 1a, b) and ANCT (Figure 1c, d) tissues. Immunoscores were calculated when the presence of eIF3f deposits in tumor glands and cytoplasmic staining were positive. The rate of high eIF3f expression in GC and ANCT tissues was $33.8 \%(66 / 195)$ and $59.5 \%(116 / 195)$, respectively.

\section{Correlation between elF3f expression and clinicopathological features}

The association of eIF3f levels with clinicopathological features in GC was also assessed in Table 1. Low eIF3f expression in GC was significantly associated with more advanced tumor stages $(P=0.02)$ and likelihood of recurrence $(P=0.04)$. However, eIF3f expression was not significantly correlated with sex $(P=0.87)$, age $(P=0.17)$, tumor differentiation $(P=0.46)$, and tumor size $(P=0.16)$. Out of 136 stage I-II cases, $53(40.0 \%)$ had high eIF3f

Table 1 Correlation among elf3f staining and clinical characteristics

\begin{tabular}{|c|c|c|c|c|}
\hline Characteristics & No. & $\begin{array}{l}\text { elF3f-high }(n= \\
66)\end{array}$ & $\begin{array}{l}\text { elF3f-low }(n= \\
129)\end{array}$ & $P$ \\
\hline \multicolumn{5}{|l|}{ Gender } \\
\hline Male & 142 & 49 & 93 & \multirow[t]{2}{*}{0.87} \\
\hline Female & 53 & 17 & 36 & \\
\hline \multicolumn{5}{|l|}{ Age (y) } \\
\hline$<60$ & 84 & 33 & 51 & \multirow[t]{2}{*}{0.17} \\
\hline$\geq 60$ & 111 & 33 & 78 & \\
\hline \multicolumn{5}{|l|}{ Tumor differentiation } \\
\hline Well & 12 & 8 & 4 & \multirow[t]{3}{*}{0.46} \\
\hline Moderate & 27 & 4 & 23 & \\
\hline Poor & 156 & 54 & 102 & \\
\hline \multicolumn{5}{|l|}{ Tumor size $(\mathrm{cm})$} \\
\hline$<4.5$ & 114 & 34 & 80 & \multirow[t]{2}{*}{0.16} \\
\hline$\geq 4.5$ & 81 & 32 & 49 & \\
\hline \multicolumn{5}{|l|}{ TNM stage } \\
\hline$|-| \mid$ & 136 & 53 & 83 & \multirow[t]{2}{*}{0.02} \\
\hline III & 59 & 13 & 46 & \\
\hline \multicolumn{5}{|l|}{ Her-2 status } \\
\hline Negative & 164 & 53 & 111 & \multirow[t]{2}{*}{0.30} \\
\hline Positive & 31 & 13 & 18 & \\
\hline \multicolumn{5}{|l|}{ Primary/Recurrent } \\
\hline Primary tumor & 116 & 46 & 70 & \multirow[t]{2}{*}{0.04} \\
\hline $\begin{array}{l}\text { Recurrent } \\
\text { tumor }\end{array}$ & 79 & 20 & 59 & \\
\hline
\end{tabular}



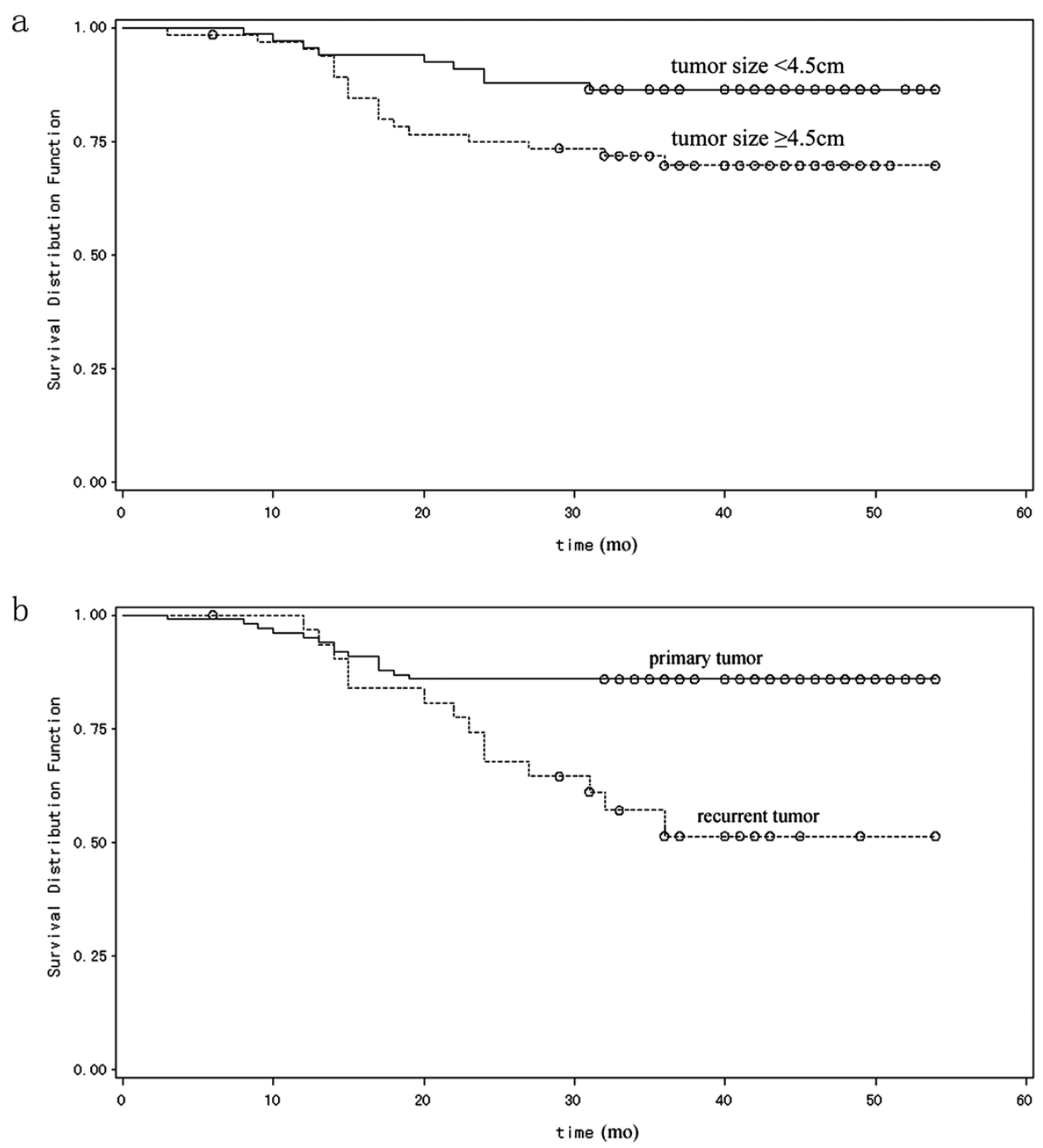

$\mathrm{C}$

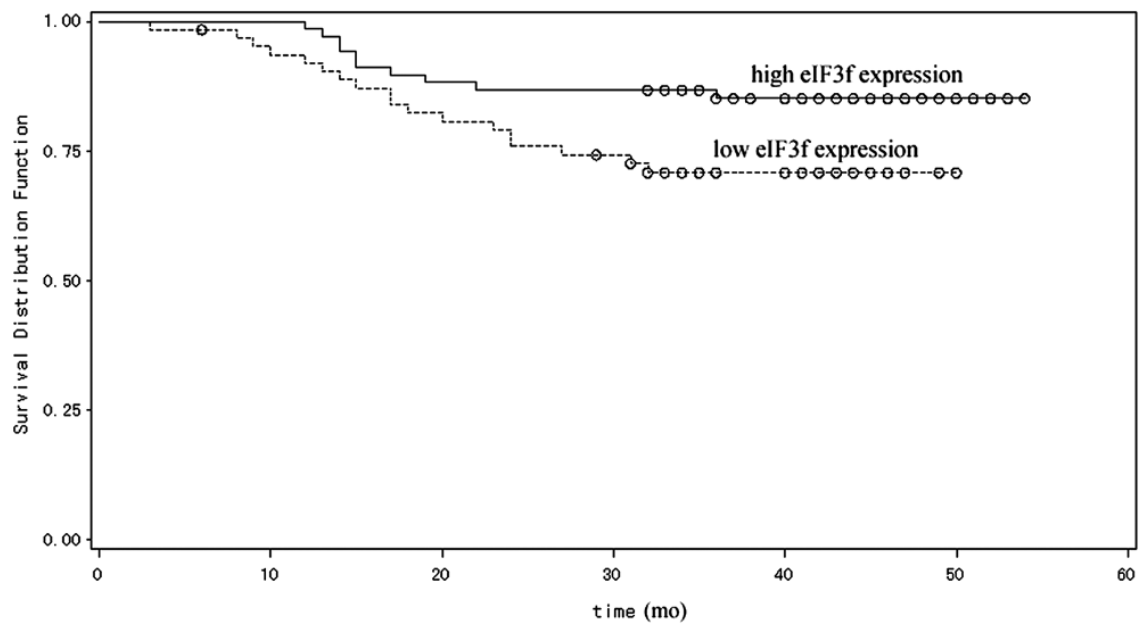

Figure 2 Overall survival curves for gastric cancer (GC) patients. (a) Kaplan-Meier overall survival curves for GC patients with tumor size $<4.5 \mathrm{~cm}$ and $\geq 4.5 \mathrm{~cm}$. GC patients with tumor size $\geq 4.5 \mathrm{~cm}$ had significantly shorter overall survival $(P=0.03)$ than those $<4.5 \mathrm{~cm}$. (b) Kaplan-Meier overall survival curves for primary or recurrent GC patients. Recurrent GC patients had significantly shorter overall survival $(P<0.01)$ than primary GC patients. (c). A comparison of the overall survival curve between patients with low and high elF3f expression. The median progression-free survival was 42.5 months in patients with high elF3f expression, compared to 33 months in patients with low elF3f expression. 
staining densitometry values, and these were higher than cases of stage III $(P=0.02)$. Furthermore, the overall densitometric analysis of immunostaining revealed that the downregulated eIF3f protein expression varied significantly between primary and recurrent tumors $(P=0.04)$.

\section{The relationship between the expression level of elF3f and prognosis}

The prognostic effect of eIF3f on the survival rate of GC patients was investigated by comparing the survival rate of patients with high or low levels of eIF3f protein expression in tumors using Kaplan-Meier survival curves and log-rank test. Univariate Cox regression analysis showed that clinical variables, including tumor size (logrank, $P=0.03$; Figure 2a), tumor recurrence (log-rank, $P<0.01$; Figure 2b), and eIF3f expression (log-rank, $P=0.04$; Figure $2 \mathrm{c}$ ) were all significantly associated with overall survival (Table 2). Data indicates that high expression of eIF3f protein was a significant prognostic factor for better survival of GC patients. Furthermore, multivariate Cox regression analyses showed that eIF3f expression was an independent predictor for the overall survival of patients with GC.

\section{Discussion}

Although the incidence and mortality rate of $\mathrm{GC}$ have fallen over the past several decades, GC is still the fourth most common cancer and the second leading cause of cancer-related death in the world [22,23]. Disappointingly, little improvement has been achieved within the past several decades despite advances in tumor diagnosis and treatment $[24,25]$. Given the high failure rate of conventional treatment strategies, many cancer-related molecules have been characterized with the goal of developing novel anticancer strategies.

eIF3f is another eIF3 subunit whose function is not well-known $[18,26]$. Previous studies have demonstrated that overexpression of eIF3f inhibits cell proliferation and induces apoptosis in melanoma and pancreatic cancer cells, suggesting that downregulation of eIF3f is involved in tumorigenesis for many types of cancer $[11,16,19]$. However, there is no large sample report about the expression and clinical significance of eIF3f in human GC progression and prognosis. In this study, we investigated the expression of eIF3f in GC as well as its correlation with the clinicopathological features and prognosis.

The data from our study showed that the levels of eIF3f protein were significantly lower in GC tissue compared with paired ANCT. Additionally, the decreased expression of eIF3f protein was significantly related to more advanced tumor stages $(P=0.02)$ and likelihood of recurrence $(P=$ $0.04)$. No statistically significant relationship was found between the eIF3f levels and sex $(P=0.87)$, age $(P=0.17)$,
Table 2 Univariate and multivariate analysis of overall survival in patients with gastric cancer

\begin{tabular}{|c|c|c|c|}
\hline & No. & $\begin{array}{l}\text { Hazard ratio } \\
(95 \% \mathrm{Cl})\end{array}$ & $P$ \\
\hline \multicolumn{4}{|l|}{ Univariate analysis } \\
\hline \multicolumn{4}{|l|}{ Gender } \\
\hline Male & 142 & & 0.40 \\
\hline Female & 53 & & \\
\hline \multicolumn{4}{|l|}{ Age (y) } \\
\hline$<60$ & 84 & & 0.12 \\
\hline$\geq 60$ & 111 & & \\
\hline \multicolumn{4}{|l|}{ Tumor differentiation } \\
\hline Well & 12 & & 0.74 \\
\hline Moderate & 27 & & \\
\hline Poor & 156 & & \\
\hline \multicolumn{4}{|l|}{ Tumor size $(\mathrm{cm})$} \\
\hline$<4.5$ & 114 & & 0.03 \\
\hline$\geq 4.5$ & 81 & & \\
\hline \multicolumn{4}{|l|}{ TNM stage } \\
\hline$|-| \mid$ & 136 & & 0.88 \\
\hline III & 59 & & \\
\hline \multicolumn{4}{|l|}{ Her-2 status } \\
\hline Negative & 164 & & 0.12 \\
\hline Positive & 31 & & \\
\hline \multicolumn{4}{|l|}{ Primary/Recurrent } \\
\hline Primary tumor & 116 & & $<0.01$ \\
\hline Recurrent tumor & 79 & & \\
\hline \multicolumn{4}{|l|}{ elF3f expression } \\
\hline Low & 129 & & 0.04 \\
\hline High & 66 & & \\
\hline \multicolumn{4}{|l|}{ Multivariate analysis } \\
\hline \multicolumn{4}{|l|}{ Tumor size $(\mathrm{cm})$} \\
\hline$<4.5$ & 114 & 2.31 & 0.04 \\
\hline$\geq 4.5$ & 81 & $1.04-5.11$ & \\
\hline \multicolumn{4}{|l|}{ Primary/Recurrent } \\
\hline Primary tumor & 116 & 3.77 & $<0.01$ \\
\hline Recurrent tumor & 79 & $1.79-7.94$ & \\
\hline \multicolumn{4}{|l|}{ elF3f expression } \\
\hline Low & 129 & 2.27 & 0.04 \\
\hline High & 66 & $1.05-4.92$ & \\
\hline
\end{tabular}

tumor differentiation $(P=0.46)$, and tumor size $(P=0.16)$. The Kaplan-Meier survival curves showed that patients with low eIF3f expression had significantly poorer survival compared with patients with high eIF3f expression. Furthermore, multivariate Cox regression analyses showed that tumor size $(P=0.04)$, recurrence $(P<0.01)$, and eIF3f 
expression $(P=0.04)$ were all prognostic predictors of human GC after resection. Based on these results, we propose a bold hypothesis that a preoperative determination of eIF3f expression may be useful in predicting the therapeutic effect and postoperative survival of human GC. A drawback of the present study is the lack of investigation of eIF3f at the molecular and cellular levels in GC. Therefore, further studies should be performed for a more concrete evidence to support the correlation between eIF3f expression and the malignant degree of GC.

\section{Conclusion}

In summary, eIF3f is downregulated in GC. Ultimately, eIF3f may play an important role in the progression and recurrence of GC. However, the specific regulatory mechanisms remain to be further studied to provide potential targets for the treatment of GC.

\section{Abbreviations \\ ANCT: Adjacent non-cancerous tissues; elF3f: Eukaryotic initiation factor $3 \mathrm{f}$; GC: Gastric carcinoma; PBS: Phosphate-buffered saline; TNM: Tumor, node, metastasis.}

\section{Competing interests}

The authors declare that they have no competing interest.

\section{Authors' contributions}

GL designed the study and gave conceptual advice; CS performed experiments; BL collected data and performed the follow-up work of all patients; NW analyzed data and wrote the manuscript. All authors read and approved the final manuscript.

\section{Author details}

'Department of Oncology, The People's Hospital of Zhangqiu, Shandong Province, 38th Huiquan Road, Jinan 250200, China. ²Department of Nephrology, The People's Hospital of Zhangqiu, Shandong Province, 38th Huiquan Road, Jinan 250200, China. ${ }^{3}$ Central Electrocardiogram Department, The People's Hospital of Zhangqiu, Shandong Province, 38th Huiquan Road, Jinan 250200, China. ${ }^{4}$ Department of General Surgery, The Second Hospital of Shandong University, 247th Beiyuan Avenue, Jinan 250200, China.

Received: 1 August 2013 Accepted: 16 March 2014

Published: 28 March 2014

\section{References}

1. Jemal A, Bray F, Center MM, Ferlay J, Ward E, Forman D: Global cancer statistics. CA Cancer J Clin 2013, 61:69-90.

2. Batista TP, Santos CA, Almeida GF: Perioperative chemotherapy in locally advanced gastric cancer. Arq Gastroenterol 2013, 50:236-242.

3. Xiao JW, Chen JH, Ren MY, Tian XB, Wang CS: Relationship between expression of gastrokine 1 and clinicopathological characteristics in gastric cancer patients. Asian Pac J Cancer Prev 2012, 13:5897-5901.

4. Xu X, Yang X, Xing C, Zhang S, Cao J: miRNA: the nemesis of gastric cancer (Review). Oncol Lett 2013, 6:631-641.

5. Juca PC, Lourenco L, Kesley R, Mello EL, Oliveira IM, Correa JH: Comparison of survival and prognostic factors in patients with gastric adenocarcinoma in T2 and T3. Rev Col Bras Cir 2012, 39(5):377-384

6. Xing $X$, Tang $Y$, Yuan G, Wang Y, Wang J, Yang Y, Chen M: The prognostic value of E-cadherin in gastric cancer: a meta-analysis. Int J Cancer 2013, 132(11):2589-2596.

7. Hu BS, Hu H, Zhu CY, Gu YL, Li JP: Overexpression of GOLPH3 is associated with poor clinical outcome in gastric cancer. Tumour Biol: J Int Soc Oncodev Biol Med 2013, 34(1):515-520.

8. Pan XF, Xie Y, Loh M, Yang SJ, Wen YY, Tian Z, Huang H, Lan H, Chen F, Soong R, Yang CX: Polymorphisms of XRCC1 and ADPRT genes and risk of noncardia gastric cancer in a Chinese population: a case-control study. Asian Pac J Cancer Prev 2012, 13(11):5637-5642.

9. Xiao $H, X u L H$, Yamada $Y$, Liu DX: Coronavirus spike protein inhibits host cell translation by interaction with elF3f. PLoS One 2008, 3(1):e1494.

10. Masutani M, Sonenberg N, Yokoyama S, Imataka H: Reconstitution reveals the functional core of mammalian elF3. EMBO J 2007, 26(14):3373-3383.

11. Shi J, Kahle A, Hershey JW, Honchak BM, Warneke JA, Leong SP, Nelson MA: Decreased expression of eukaryotic initiation factor $3 \mathrm{f}$ deregulates translation and apoptosis in tumor cells. Oncogene 2006, 25(35):4923-4936.

12. Wen F, Zhou R, Shen A, Choi A, Uribe D, Shi J: The tumor suppressive role of elF3f and its function in translation inhibition and rRNA degradation. PLoS One 2012, 7(3):e34194.

13. Moretti J, Chastagner P, Gastaldello S, Heuss SF, Dirac AM, Bernards R, Masucci MG, Israel A, Brou C: The translation initiation factor $3 f$ (elF3f) exhibits a deubiquitinase activity regulating Notch activation. PLOS Biol 2010, 8(11):e1000545.

14. Jiang B, Zhang B, Liang P, Song J, Deng H, Tu Z, Deng G, Xiao X: Nucleolin/ C23 mediates the antiapoptotic effect of heat shock protein 70 during oxidative stress. FEBS J 2010, 277(3):642-652.

15. Ray A, Bandyopadhyay A, Matsumoto T, Deng $H$, Maitra U: Fission yeast translation initiation factor 3 subunit elF3h is not essential for global translation initiation, but deletion of eif $3 \mathrm{~h}+$ affects spore formation. Yeast 2008, 25(11):809-823.

16. Doldan A, Chandramouli A, Shanas R, Bhattacharyya A, Leong SP, Nelson MA, Shi J: Loss of the eukaryotic initiation factor $3 f$ in melanoma. Mol Carcinog 2008, 47(10):806-813.

17. Marchione R, Leibovitch SA, Lenormand JL: The translational factor elF3f: the ambivalent elF3 subunit. Cell Mol Life Sci 2013, 70:3603-3616.

18. Shi J, Hershey JW, Nelson MA: Phosphorylation of the eukaryotic initiation factor $3 f$ by cyclin-dependent kinase 11 during apoptosis. FEBS Lett 2009, 583(6):971-977.

19. Doldan A, Chandramouli A, Shanas R, Bhattacharyya A, Cunningham JT, Nelson MA, Shi J: Loss of the eukaryotic initiation factor $3 \mathrm{f}$ in pancreatic cancer. Mol Carcinog 2008, 47(3):235-244.

20. Higareda-Mendoza AE, Pardo-Galvan MA: Expression of human eukaryotic initiation factor $3 f$ oscillates with cell cycle in A549 cells and is essential for cell viability. Cell Division 2010, 5:10.

21. Kwon SJ: Evaluation of the 7th UICC TNM staging system of gastric cancer. J Gastric Cancer 2011, 11(2):78-85.

22. Hadi R, Mohanti BK, Pathy S, Rath GK, Shukla NK, Deo SV, Sharma A, Raina V: Gastric cancer: a retrospective analysis from AllMS, New Delhi. Gulf J Oncolog 2012, 1(12):11-16.

23. Zagouri F, Papadimitriou CA, Dimopoulos MA, Pectasides D: Molecularly targeted therapies in unresectable-metastatic gastric cancer: a systematic review. Cancer Treat Rev 2011, 37(8):599-610.

24. Fujitani K, Yamada M, Hirao M, Kurokawa Y, Tsujinaka T: Optimal indications of surgical palliation for incurable advanced gastric cancer presenting with malignant gastrointestinal obstruction. Gastric Cancer 2011 14(4):353-359

25. D'Ugo D, Persiani R, Zoccali M, Cananzi F, Vigorita V, Mazzeo P, Tufo A, Biondi A: Surgical issues after neoadjuvant treatment for gastric cancer. Eur Rev Med Pharmacol Sci 2010, 14(4):315-319.

26. Valente ST, Gilmartin GM, Mott C, Falkard B, Goff SP: Inhibition of HIV-1 replication by elF3f. Proc Natl Acad Sci USA 2009, 106(11):4071-4078.

doi:10.1186/1477-7819-12-72

Cite this article as: Li et al: Decreased expression of eukaryotic initiation factor $3 \mathrm{f}$ is an adverse prognostic factor for stage I-III gastric cancer. World Journal of Surgical Oncology 2014 12:72. 